\title{
Ethics and programmatic issues on the 2003 SARS epidemic: are any lessons transferable to the HIV pandemic?
}

\author{
A.S. MUULA* \\ Department of Epidemiology, University of North Carolina, 529 Hillsborough St, H 7, Chapel Hill, NC 27514, \\ United States and Division of Community Health, University of Malawi, College of Medicine, Malawi
}

\begin{abstract}
The HIV / AIDS pandemic continues to spread globally but much more so in the less industrialised countries of southern and eastern Africa. Despite the difference in aetiology and modes of spread of the Severe Acute Respiratory Syndrome (SARS), there are certainly some lessons that policy makers can reflect on regarding the global control of the SARS epidemic in 2003. The response to the SARS outbreak garnering the support of every stakeholder than could be mobilized is an example of how emerging infectious diseases could be dealt with. However, the programmatic and ethics issues, although resulting in an effective response were nonetheless controversial in many ways, as the potentially compromised people's rights and autonomy. These issues require further reflection and an assessment as to whether they could be used in the fight against HIV and AIDS. Presented in this paper, are selected HIV and SARS prevention and control activities that can be assessed for potential effectiveness, ethics rating and programmatic challenges. In this article, the similarities in prevention and control are presented, so will the differences.
\end{abstract}

Key words: ethics, lessons, SARS, HIV / AIDS

\section{Introduction}

The global response to the severe acute respiratory syndrome (SARS) pandemic in 2003 (Chung et al., 2005; Tapper, 2006) have raised important ethical and programmatic considerations especially with regard to balancing personal freedoms and at the same time ensuring that public health is enhanced. Many public health professionals and the World Health Organization report of great satisfaction as to how what could have been a worse pandemic was dealt with in a collaborative and decisive manner that within a few months, SARS was no longer a public health threat that it posed to be.

Isolation and quarantine, restriction of travel was used to prevent transmission and acquisition of SARS while mandatory clinical examinations were conducted among those potentially exposed and susceptible persons. In this paper, lessons learnt from the SARS pandemic will be suggested for HIV prevention and control. While the world community took decisive and in many situations controversial decisions, the same has not always been possible with HIV, and lack of decisive action has, in part been responsible for the unrelenting spread of HIV. While the respect of human rights and ethical codes is an important attribute in the health services sector, the lessons so far obtained from the SARS epidemic could guide future ethics debate in HIV prevention, care and support.

\section{Africa and the HIV pandemic}

While no continent has been spared by the HIV / AIDS pandemic, Africa, especially southern Africa, continues to be the hardest hit region (Muula, 2004). It is important to make this distinction that it is southern Africa, not the whole of Africa that has suffered the brunt of the epidemic. North Africa, politically described as if not part of the continent is home to only a small proportion of people infected by HIV. Although West Africa has been affected, the numbers and proportion of the population infected is no where near that of eastern and southern Africa. The reasons for this difference have been suggested and include religion, poverty, culture and economic differences. West Africa also seems to be affected by the less virulent HIV-2 as opposed to HIV-1, which is predominant in eastern and Southern Africa. It is however of interest that by January 2006, at least two countries in this hardest-affected region were registering a drop in the incidence of HIV. These countries are Uganda and Zimbabwe and lessons learnt from these two countries are being awaited to see if they could result in incidence drops in other highly affected nations.

\section{SARS versus HIV/AIDS}

In 2003, an emerging disease the severe acute respiratory syndrome (SARS) was reported in Hong Kong, China and within a short period, in many

\footnotetext{
* Address correspondence to: Dr. Adamson S. Muula, Email: muula@email.unc.edu
} 
parts of the world. Tourism and the airline industry were particularly affected as international travel was curtailed significantly and countries affected by SARS were avoided by travellers (Venkatesh \& Memish, 2004). The response to the outbreak garnering the support of every stakeholder than could be mobilized is an example of how emerging infectious diseases could be dealt with. The programmatic and ethics issues, although resulting in an effective response were nonetheless controversial in many ways, as the potentially compromised people's rights and autonomy. These issues require further reflection and an assessment as to whether they could be used in the fight against HIV and AIDS. Presented below are selected HIV and SARS prevention and control activities that will be assessed for potential effectiveness, ethics rating and programmatic challenges.

\section{Prompt case detection}

Much of the prevention of SARS transmission was made because of a highly effective system of case detection. It was because of case detection that various prevention and treatment efforts such as isolation and quarantine could be instituted. In the absence of specific clinical features, sensitivity was the main concern with specificity not so much of a concern. It was important at the time to identify as many infected persons as possible. Whether someone would be wrongly identified, as a SARS case when they were not, was not much of a problem.

In dealing with HIV, we are yet to be concerned with identification of recent infection. Although ongoing research in diagnostics seems to be promising and in the next few years tests to identify acute HIV infection would be in routine use, it is somewhat unclear as to whether these tests would be made routinely available especially in the highly affected areas of the world such as Africa. While in many developed nations one can envisage that partner notification, and in many developed nations, this is mandatory, in many African nations, partner notification is left to the index case. Public health systems even do seem to be ambiguous regarding partner notification as there are concerns about stigma, discrimination and marital discord when disclosure of HIV sero-positive status is made.

It is noteworthy that during the SARS pandemic, many countries severely affected enacted legislation, which made not disclosure of possibility of SARS infection, a criminal offence. In Singapore, for example, the Infectious Disease Act (James et al., 2006) of stated that it was an individual's responsibility to: go to a designated treatment site if one had an infectious disease; not to go to public places if suspecting infection; and no breaking of home quarantines. There was also the possibility of electronic tagging and forced adherence for violators; destruction of suspected contaminated fomites to be quarantined; home monitoring of body temperature and seeking medical attention upon occurrence any clinical features suggestive of SARS; and penalty of about $\$ 5800$ and six months in prison.

The prevention and control of SARS using these methods was made possible because the disease is an acute illness. A recently infected individual demonstrate clinical features (although with relatively poor specificity) within a short time. Also, although no known cure has been found, people can get cured and do not seem to remain infected forever. There is also no concern of infectiousness once the clinical syndrome is through.

\section{Quarantine and isolation}

Quarantine and isolation are age-known effective public health intervention in the prevention and control of infectious diseases. As SARS is mostly spread through aerosol from human to human, it was important that those infected be prevented from contact with susceptible individuals. Quarantine and isolation was not just recommended but enforced, and in some cases, by use of what can be described as untraditional means. In Singapore, for example, a security firm was hired to enforce quarantined and electronic (Epic) cameras were installed in homes to monitor movements. The military was called to enforce quarantine in Taiwan.

The same principles of quarantine and isolation could be applied to HIV infection prevention, but in a modified way, but with enforcement of legislation. The use of security forces and cameras is certainly not appropriate but some legal changes are certainly necessary.

HIV and SARS are not transmitted in the same way. While SARS is mostly spread through aerosol, sexual transmission is the main mode of HIV transmission in Africa. Although rape and other sexual crimes are increasingly been reported and certainly contribute to the spread of HIV, most of the transmission of HIV is through consensual sex.

Consider an individual who knows she/ he is HIV infected. As it should be, this person is not obliged to tell anyone about his or her HIV status. The person is not even obligated to tell her sexual partner(s) about the HIV infection. It all depends on what she/he thinks her/himself, to tell or not to tell. This same person may then engage in sexual 
relations with new partners. She/he is not obligated by law to inform anyone whether she is infected or not. Her/her partner(s) before she/he tested positive, who may or may not be HIV infected, may also get new partners. Because these may not know they are infected (and even if they knew they would not have told anyone) they engage in unprotected sexual intercourse therefore potentially spread the infection. There is need for African to consider seriously legislating such that HIV infected vaginal, anal and seminal fluid is 'quarantined' or 'isolated'. This does not mean that HIV infected person should not have sexual relations. No. What is being proposed is that any person who knows they are HIV infected should be obligated to inform sexual partner(s) before any unprotected sexual relations occur. Anything less than that is gross irresponsibility. HIV infected persons should therefore ensure that protected sexual intercourse is the norm.

There is certainly one school of thought that suggest that public health re-enforcement of regulation should only occur when the person at risk of HIV infection does not have control of being exposed. This would work in case of second-hand tobacco exposure (passive smoking) when the second person is being exposed to a common environment that has been 'polluted' by the person smoking. Similarly, in the case of SARS, where the diseases-causing agent is excreted, although not voluntarily, into the environment by a patient, it can be argued that one does not really have a choice as to who should they come into contact with in normal daily activities. In contrast with HIV, most of the sexual activity is deemed consensual and it is argued that anyone should know that sexual intercourse has attendant risks, one of which is HIV.

While the above situation is presented, it is also important to recognise that while it is incumbent upon every individual to realise the dangers of sex, it can be considered a special case where an individuals knows he or she is HIV infected, the partner does not know that, but the infected partner is not constrained by any regulations to inform the other partner. There are certainly problems with enforcement with any such regulations.

\section{Contact tracing}

Effective contact tracing is an inevitable aspect on any sexually transmitted infection (STI) treatment and control programme. Between 1950 and1990s, syphilis was a major public health problem in the United States. That is why the country had the Tuskegee scandal (White, 2004). However, the situation in the 21st century is significantly different. Other than in selected 'core' communities, syphilis is no longer a major public health threat; most probably as a result of contact tracing.

Contact tracing continues to be a problem in most parts on Africa. The reasons for this are diverse but include limited human and financial resources and other operational difficulties that make communication difficult. This does not however mean that it should not be pursued but rather that its operation may have to be adapted to local realities.

\section{Voluntary counselling and testing}

The literature on HIV voluntary counselling and testing (VCT) is incomplete. Acceptability of VCT, availability and access issues and the use of it to test people in research setting has been documented (Leichty, 2005; Manzi et al., 2005). What seems to be lacking is how VCT has been used in contact tracing of persons who may be susceptible to infection. It is appropriate to have people come for VCT, but if no contact tracing is conducted, it may not be sufficient to leave the individual to their own will to disclose possibility of HIV infection to their sexual partner(s). It is indeed true that individuals have suffered violence and neglect upon disclosure of HIV status, but is also very difficult to continue in this veil of secrecy if HIV is to be controlled in Africa.

The limitation of VCT is such that there may be a large temporal distance between time of exposure, time of conversion of sero-positivity and time for HIV testing. This therefore limits the utility of contact tracing where the time of testing and the time of exposure has been separated by a huge time gap. A person may be exposed to HIV about a decade ago, may have had other sexual period in the intervening period and only have testing after having sexual intercourse with many other partners. While obviously, any of these sexual partners may have been exposed and may potentially benefit from the information of HIV sero-positivity of the index case, it poses a programmatic challenge.

While the situation above may not be uncommon but is complex, other situations may not be as complicated. There are persons who test positive, have known recent partners but then are not encouraged to reveal their HIV status to any of 
their immediate sexual partners so as to encourage them also to go for HIV test and inform their own other sexual partners about possibility of HIV infection. In many situations, HIV testing is thought of at the individual level.

\section{HIV demands action}

In 1998 at a tertiary hospital in Malawi, a very sick paediatric patient was diagnosed with Pneumocystis jiroveci pneumonia (Graham, 2005). The treatment care team had discussed the possibility of HIV infection with the grandmother but not with the mother of the patient. HIV testing of the patient was done and it was confirmed that she was HIV infected. Unlike the situation from 2004, there was no anti-retroviral therapy at the time in public health facilities in the country. Within a few days, the patient died. In the coming years, the family had two more children, one of whom died and the other was constantly sick. At this time HIV testing of the mother was done and she was diagnosed HIV infected. As she was sick herself and meeting clinical criteria for antiretroviral therapy (ART), she was put on treatment.

On one hand the decision not to inform the mother about the HIV infection of her own daughter could be justified. She was already worried about the lack of positive progress in her daughter's medical condition. People around her did not want her to give up hope. It is not known what would have happened to her marriage if the husband had known that the daughter had HIV. She may have been blamed for the infection. She may have suffered violence and/or divorce.

While the above justifications for not telling her are understandable, there are also other issues that we need to consider. Would the family have gone ahead having two more children? While several years back we wanted to protect the woman's marriage through non-disclosure, the marriage did not end and we had no reports she suffered violence when she was diagnosed herself and she was put on ART. Of course it is problematic to assume that what could have happened in 1998 could also have happened several years later.

Some clinicians have suggested that in situations as reported above, the clinical care team had obligation to report the HIV testing to the parents and not the grandmother and the parents had the onus either to tell or not anyone else including the grandmother. Other health practitioners have taken the communitarian approach where they have argued that a child does not just belong to his or her parents but rather that the extended family has various roles to play. Due to cultural differences and local situations, it can become difficult for clinicians to determine the limits of nuclear family autonomy as opposed to the communitarian aspects of the extended family.

HIV infection thrives in environments where fear abounds. Fear of disclosure. Fear of offending one's sexual partner if you ask for a condom or HIV test before marriage. Fear of testing in case one tests positive. Fear of discrimination. Fear of stigma. Fear of broken marriages. Fear of poverty if the woman is divorced. Fear of losing friends. Fear of initiating depression should a person know they are HIV infected. Fear of dying fast due to worries if found infected. Fear of loss of love.

While there is no doubt that the financial resources raised internationally by the Global Fund, Bill and Melinda Gates Foundation (Mozynski, 2005) and the commitment from the highly affected countries are crucial to the stemming of HIV, we have not so much cared in investing in society in such a way that we deal decisively with the fears mentioned above. If women's livelihoods are inextricably reliant on their being providers of sexual favours, then it becomes extremely difficult for them to avoid situations that put them at risk of HIV. If people who disclose about their being HIV infected are discriminated against, it becomes difficult to encourage HIV testing. Despite the abundance of literature on the presence of discrimination, there is paucity of data as to how this discrimination is being faced head on. Who has been indicted to appear in court for discriminating against an HIV infected person at place of work?

\section{Visitation restriction}

During the SARS pandemic, restriction of visitation was enforced. In some setting, relatives and friends of patients were totally excluded from visiting while in some cases, visits were facilitated by videoconferencing (Ovadia et al., 2005; Rogers, 2004). Considering how HIV is spread, visitation of patients admitted in hospitals may not be a major problem as HIV is very unlikely to be transmitted through casual contact. But it is important to realize that AIDS patients have an immuno-compromising situation that predisposes them to acquire a host of other infections. Some visitations to HIV infected person can be sources of infection.

HIV and SARS are different diseases in the way that while SARS patients are potentially excreting the disease-causing organism in respiratory fluid and this will be released to the general environment, HIV infected person have potential to spread infection only where there is direct contact of body fluids via sexual intercourse, blood contact in injecting instruments or contact with infected fluid on open sores. HIV infected 
individuals are not infectious in normal social contact while SARS patients are infectious in normal social contact.

In as far as visitor restriction is concerned, HIV infected person may be prevented from having certain or many visitors in order to prevent the HIV infected person from catching infection. This can be compared to situations where a cancer patient on chemotherapy, or patients with any other form of acquired immuno-suppression is isolated from potentially infectious situations, including many visitors. In the case of HIV, the potential for misunderstanding is huge such that concerns about segregation are real.

\section{Should HIV be a reportable infection?}

The issue as to whether to make or keep HIV as a reportable infection deserves serious consideration. There are countries in the world where it is mandatory to report all HIV infections. In some other countries, reports of HIV infection tests done and numbers of positive tests are reported to national HIV/AIDS programmes. This is not strictly speaking, being 'reportable'. Reportable medical conditions imply that a person's identity and other demographic details are reported to authorities to spur action. As results of how HIV infected persons were dealt with at the start of the pandemic in the early 1980s, many countries do not report names of people infected by HIV to authorities. It is also not known if such actions would prevent people from VCT. Making HIV a reportable infection can be both good and undesirable. As for the positive aspects that may arise from reporting HIV include: First, ensuring better estimates of people infected with HIV. In many African countries, it is possible to access VCT without having one's identity recorded. This, it is suggested, ensures confidentiality. However, in the end what one has are numbers and figures that are impossible to know how many times the same individual has been captured. Secondly, reporting HIV infection can be linked to treatment. While countries have estimates of HIV infected persons who may require ART, this work may be made easier if it is known who is receiving therapy and who is not. For those that may not be on treatment, follow-up can be initiated, especially if their CD4 counts are known to be low. CD4 counts determination can be done from the same sample that was taken to determine HIV infection.

Not all health practitioners agree that HIV should be a reportable case, in as far as having the name and details of the infected person recorded. It is argued that it is not so much as the name that is important but rather the demographic details that are obtained from the exercise that will be used for policy decisions.

\section{Conclusion}

Public health sometimes requires that personal liberties be restrained. There continues to be hope for Africa as a few countries have started to register small but noticeable drops in incidence of HIV (Gregson et al., 2006). African countries needs to consider seriously issues regarding disclosure of HIV to people who may be at risk of infection if they do not know about an index case's HIV infection, strengthen contact tracing as ART roll-out catches speed. Disclosure of HIV status, just like disclosure of any other medical condition to any third person need to be done in the best interest of the patient or at least in the interest of public health. It should be noted that the only purpose for which power can rightfully be exercised over any member of a civilised community, against his will, is to prevent harm to others.

\section{Acknowledgements}

I am grateful to the two anonymous reviewers of the manuscript.

\section{References}

Chung, B.P., Wong, T.K., Suen, E.S. \& Chung, J.W. (2005) SARS: caring for patients in Hon Kong. Journal of Clinical Nursing 14, 410-417.

Graham, S.M. (2005) Non-tuberculous opportunistic infections and other lung diseases in HIV-infected infants and children. International Journal of Tuberculosis and Lung Disease 9, 592-602.

Gregson, S., Garnett, G.P., Nyamukapa, C.A., Hallett, T.B., Lewis, J.J., Mason, P.R., Chandiwana, S.K. \& Anderson, R.M. (2006) HIV decline associated with behaviour change in eastern Zimbabwe. Science 311, 664-666.

James, L., Shindo, N., Cutter, J., Ma, S., Chew, S.K. (2006) Public health measures implemented during the SARS outbreak in Singapore 2003. Public Health 120, 20-26.

Leichty, C.A. (2005) The evolving role of HIV counselling and testing in resource-limited settings: HIV prevention and linkage to expanding HIV care access. Current Infectious Disease Report 7, 154-158

Manzi, M., Zachariah, R., Teck, R., Buhendwa, L., Kazima, J., Bakali, E., Firmenich, P. \& Humblet, P. (2005) High acceptability of voluntary counselling and HIV-testing but unacceptable loss to follow up in a prevention of mother-to-child HIV 
transmission programme in rural Malawi: scaling-up requires a different way of acting. Tropical Medicine and International Health 10, 1042-1050.

Mozynski, P. (2005) Global fund calls for increased funding. British Medical Journal 331, 533.

Muula, A.S. (2004) Ethical and programmatic challenges in antiretroviral scaling-up in Malawi: challenges in meeting the World Health Organization's "Treating 3 million by 2005" initiative goals. Croatian Medical Journal $45,415-421$.

Ovadia, K.L., Gazit, I., Silner, D. \& Kagan, I. (2005) Better late than never: a re-examination of ethical dilemmas in coping with severe acute respiratory syndrome. Journal of Hospital Infection 61, 75-79.
Rogers, S. (2004) Why can't I visit: the ethics of visitation restrictions-lessons learned from SARS. Critical Care 8, 300-302

Tapper, M.L. (2006) Emerging viral diseases and infectious disease risk. Haemophilia 12, suppl:13-17.

Venkatesh, S. \& Memish, Z.A. (2004) SARS: The new challenge to international health and travel. East Mediterranean Health Journal 10, 655-52.

White, R.M. (2006) Effects of untreated syphilis in the Negro male, 1932 to 1972: a closure comes to the Tuskegee study, 2004. Urology 67, 654. 